\title{
Consentimiento informado para anestesia regional, bloqueos periféricos y analgesia regional ambulatoria.
}

Informed consent for regional anesthesia, peripheral blocks and ambulatory regional analgesia. https://doi.org/10.25237/carsach2020.04

Dra. Rousmery Atton M. ${ }^{1}$ Dr. Gonzalo Mutizabal M. ${ }^{2}$

1 Departamento de Anestesiología y Medicina de Dolor. Clínica Las Condes, Unidad de Anestesiología Hospital Barros Luco Trudeau. ${ }^{2}$ Servicio de Anestesiología Clínica Indisa, Servicio de Anestesiología RedSalud Vitacura

Autor Corresponsal:

Dra. Rousmery Atton M.

Departamento de Anestesiología y Medicina de Dolor. Clínica Las Condes, Unidad de Anestesiología Hospital Barros Luco Trudeau.

Dirección: Lo Fontecilla 441, Las Condes

Teléfono: 995090428, 222105251

e-mail: rousatton@gmail.com

ORCID ID: https://orcid.org/0000-0003-3927-1847

Palabras claves: Consentimiento informado, Anestesia regional, covid-19.

Key words: Informed consent, regional anesthesia, covid-19.

\section{Puntos clave}

- El proceso del CI pretende informar de buena manera a cada paciente, para que pueda tomar las mejores opciones sanitarias para sus convicciones sobre la vida que pretende vivir y los riesgos que prefiere aceptar o rechazar.

- Eso implica informar y discutir activamente los riesgos, beneficios y alternativas de tratamiento con el paciente y documentar su decisión en un formulario adecuado, con fines médico legales.

- Este proceso se basa en el principio de autonomía; capacidad del paciente de tomar decisiones y consentir y/o autorizar una determinada intervención de manera informada.

- El CI es un proceso que puede ser plasmado en un documento, no es el mero trámite de poner firmas en un documento.

- Existe variabilidad en la cantidad y calidad de información entregada al paciente. Tener un documento estándar y específico para AR podría asegurar que se discutan los riesgos más importantes de un bloqueo regional.

\section{Resumen}


El consentimiento informado (CI) no es sólo la firma de un formulario, es una obligación ética y legal que incluye el deber de informar y discutir activamente los riesgos, beneficios y alternativas de un procedimiento con el paciente. Este proceso suele ser verbal, pero en ciertos casos debe quedar documentado en formularios específicos. El cumplimiento de este proceso, y la construcción de una relación médico paciente sólida, conforman la piedra angular del cuidado del paciente y podría ayudar ante una eventual defensa médico legal.

La reactivación de la actividad quirúrgica en período de pandemia por Covid-19 considera priorizar cirugía ambulatoria y/o de corta estadía intrahospitalaria. El manejo anestésico con técnicas regionales ofrece la posibilidad de disminuir los tiempos de hospitalización.

El objetivo de esta revisión es proponer un proceso y formulario de CI específico y de calidad para anestesia regional (AR), que contenga información estándar para ser discutida con el paciente.

\begin{abstract}
The informed consent (CI) is not just the signing of a form, it is an ethical and legal obligation that includes the duty to inform and actively discuss the risks, benefits, and alternatives of a procedure with the patient. This process is usually verbal, but in certain cases, it must be documented on specific forms. Compliance with this process, together with the construction of a solid physician-patient relation, forms the cornerstone of patient care and could help with an eventual medicolegal issue.

The reactivation of surgical activity in the period of the Covid-19 pandemic considers prioritizing outpatient and / or short-stay surgery. In this context, anesthetic management with regional techniques offers the possibility of reducing hospitalization times and to procure outpatient pain management.

The objective of this review is to propose a specific and quality CI process and form for regional anesthesia (RA), which contains standard information to be discussed with the patient.
\end{abstract}

\title{
Introducción
}

El ejercicio de la medicina pretende servir a las personas modificando sus condiciones de salud, y es una actividad que suele gozar de alta estima en las sociedades donde se inserta, por la tremenda relevancia que esas condiciones de salud pueden tener para todos los ciudadanos.

En tal escenario, los médicos adquieren una especial posición frente al resto de la comunidad, ciertos derechos y deberes muy especiales (el profesionalismo), que hacen necesario ajustar sus conductas a ciertas pautas que se han ido modificando en la historia, y que se deben adaptar a las circunstancias. Un quehacer médico que se mantiene imperturbable en el tiempo o ajeno a las contingencias de la vida real podría llevar a la ejecución de prácticas poco profesionales, que pueden ser inaceptables para la comunidad, y generar sanciones éticas o judiciales.

Debido a la pandemia provocada por el virus SARS COV-2 (COVID-19) declarada por la Organización Mundial de la Salud (OMS) en marzo del 2020(1), la actividad médico-quirúrgica electiva se vio reducida drásticamente o definitivamente suspendida durante los meses en que el número de casos activos y graves de la enfermedad estresaron el sistema sanitario de nuestro país. Actualmente, considerando el curso y evolución a la baja sostenida del número de casos nuevos y la menor ocupación de camas críticas, la tendencia es ir reactivando la actividad quirúrgica. Para ello se han planteado protocolos ministeriales y de sociedades médicas, entre ellas la Sociedad Chilena de Anestesiología $(\mathrm{SACH})$ de reinicio seguro de la actividad médica(2,3). Estos documentos están orientados a brindar la máxima seguridad a los pacientes y al personal de salud en este nuevo escenario en que la persistencia de la enfermedad por COVID19 sigue afectando a nuestra población y el riesgo de contagio permanece latente.

Los protocolos de reinicio seguro de la actividad quirúrgica condicionan el tipo de cirugía a aquellas electivas necesarias sensibles al tiempo (MeNTS), de estadía intrahospitalaria corta y cirugía ambulatoria mayor. En este contexto, la técnica anestésica y analgésica cobra especial relevancia al acelerar la rehabilitación del paciente y permitir el alta temprana. 
Las recomendaciones de expertos sugieren preferir anestesia regional (AR) por sobre anestesia general $(3,4)$. Tanto la AR central como periférica tienen mejor relación riesgo/beneficio(3), con una rápida recuperación y alta hospitalaria precoz.

En este contexto, protocolizar procesos perioperatorios como por ejemplo el consentimiento informado, es perentorio en la "Era Covid". Esta es la oportunidad de desarrollar procesos e instrumentos que permitan realizar técnicas de AR ambulatorias y domiciliarias con respaldo médico legal así como también objetivar y evidenciar que la información y educación que se entrega al paciente fue entendida y los riesgos aceptados.

Esta revisión pretende proponer una estructura del proceso y formulario de consentimiento informado de buena calidad para AR, definiendo efectos adversos y riesgos relacionados al procedimiento, que asegure una discusión informada respetando la autonomía del paciente y una adecuada documentación y/o registro de ello.

\section{Metodología de revisión}

Se realizó una revisión narrativa de artículos relacionados en Pubmed. Se utilizaron los términos Mesh: Informed consent, Nerve blocks, Anesthesia and Analgesia, Covid-19, incluyéndose artículos en inglés y español.

\section{Historia}

Visiones más completas de esta materia están disponibles en los archivos de la Revista Chilena de Anestesiología, tanto para el ejercicio clínico como para la investigación en medicina $(5,6)$. La expresión "consentimiento informado" puede hacer referencia a varios conceptos diferentes: un proceso sanitario, un requisito administrativo, un derecho inalienable y personalísimo del paciente, un mero formulario, un hecho controvertido que se debe demostrar en juicio, etc.

En términos históricos es un concepto generado fuera del mundo médico, específicamente en el mundo jurídico de las culturas británica (los primeros esbozos) y norteamericana (su definición más madura).

Durante mucho tiempo el ejercicio médico fue más bien contemplativo, casi un acompañamiento pasivo, capaz de ofrecer escasas medidas paliativas y cierto grado de información y pronóstico en tiempos de enfermedad. El lado virtuoso de esa limitada posición es que las escasas intervenciones disponibles estaban amparadas por un alto nivel de inocuidad (toda clase de infusiones y cataplasmas) o de necesidad (amputaciones).

En tiempos recientes aparecieron intervenciones mucho más eficientes: fármacos (algunos muy peligrosos), radiaciones, cirugía, anestesia, etc. Pero, ese explosivo desarrollo de la efíciencia ha ido aparejado a un explosivo aumento en el nivel de riesgo de muchas intervenciones médicas, incluso aquellas que son perfectamente bien indicadas y ejecutadas (tomando la anafilaxia mortal como el ejemplo más extremo).

Por eso la comunidad de potenciales pacientes ha ido reclamando el derecho a decidir las intervenciones que acepta para su cuerpo, porque nada ni nadie puede garantizar el éxito de ninguna de ellas, y porque en un Estado democrático, el bienestar de cada quien lo decide cada cual. Además, hay situaciones especiales, como la interpretación textual de la Biblia que subyace al rechazo de hemoderivados, que es frecuente en los Testigos de Jehová (un punto claramente relacionado con el derecho a la libertad de culto); el derecho a decidir de manera autónoma sobre la propia vida y la propia muerte, en la eutanasia (una manifestación de que todos los humanos nacen libres e iguales en dignidad y derechos); y el derecho a aceptar o postergar del derecho a la reproducción, en la interrupción voluntaria del embarazo (un recordatorio vigente de que algunos Estados han llegado a perturbar hasta las más íntimas y privadas manifestaciones de la autonomía individual).

El origen y mayor desarrollo de esta doctrina en el hemisferio norte, en el mundo del derecho británico y norteamericano, se puede atribuir a dos factores fundamentales:

1.- $\mathrm{Su}$ cultura protestante, que valora en grado extremo el respeto al otro y el valor del contrato entre iguales, donde el cumplimiento de la palabra empeñada se considera un valor en sí mismo, una forma de cercanía con la divinidad.

2.- Su estructura judicial basada en el derecho consuetudinario, en que las sentencias se ajustan de manera más dinámica a los cambios observados en la vida de la comunidad, sin necesidad de esperar la dictación de leyes lentas y farragosas, que suelen ir atrasadas con respecto a los hechos de la realidad.

Una breve enumeración de hitos judiciales debe incluir algunos casos clásicos, disponibles en internet:

Slater v. Baker \& Stapleton (1767). 
Exige el consentimiento del paciente en cuanto forma parte del hacer habitual de los cirujanos. No menciona la necesidad humanista, ética o jurídica de obtener el consentimiento del paciente antes de intervenirlo. Sólo invoca una costumbre profesional que probablemente se debía a que las intervenciones se realizaban sin anestesia y, por ello, era muy deseable contar con la cooperación, más o menos estoica, del que se operaba.

Mohr v. Williams (1905).

Proscribe la costumbre médica de tomar decisiones "técnicamente razonables", como operar primero el oído más enfermo. El tribunal estableció que el consentimiento de la paciente se refería específicamente al oído derecho, y que había sido incorrecto extenderlo por analogía al lado izquierdo, porque la cirugía del lado izquierdo no era una urgencia y no había sido consentida.

Pratt vs Davis (1906).

El cirujano hizo una histerectomía no autorizada, y afirmó en su defensa que: “... Cuando una paciente se pone al cuidado de un cirujano para tratamiento sin limitaciones expresas, bajo su autoridad, ella consiente legalmente que él puede realizar toda operación que considere apropiada y esencial para el bienestar de ella".

El fallo fue demoledor y debería ser conocido por todo médico:

- "[Under a free government at least, the free citizen's first and greatest right, which underlies all others -the right to the inviolability of his person, in other words, the right to himself-is the subject of universal acquiescence, and this right necessarily forbids a physician or surgeon, however skillful or eminent $[\ldots]$ to violate without permission the bodily integrity of his patient [...] and [to operate] on him without his consent or knowledge".

Mary E. Schloendorff v. The Society of the New York Hospital (1914).

Es el punto inicial de la doctrina moderna del consentimiento informado, porque en él se hace un reproche jurídico al quehacer sanitario bienintencionado, en que se toman decisiones aparentemente razonables (hacer un examen bajo anestesia general, y extirpar el tumor que se hace evidente); pero, "contrarias a la voluntad expresa del afectado" (que había prohibido expresamente esa eventual cirugía).

- "In the case at hand, the wrong complained of is not merely negligence. It is trespass. Every human being of adult years and sound mind has a right to determine what shall be done with his own body; and a surgeon who performs an operation without his patient's consent commits an assault, for which he is liable in damages".

Es necesario saber que "assault" (amenaza o riesgo de agresión física) y "battery" (agresión física) son ilegales en la vida cotidiana, y el operador sanitario sólo tiene derecho a ejecutarlas cuando tiene un consentimiento informado y actúa de acuerdo a la lex artis ad hoc. La señora Schloendorff perdió el juicio y fue condenada a pagar 292 dólares de costas, porque se consideró que había demandado a una institución de beneficencia. Pero, el legado del derecho a decidir sobre el propio cuerpo sigue vigente más de un siglo después de los hechos.

Salgo v. Leland Stanford Jr. University Board of Trustees (1957).

Ese fallo consolidó los dos elementos fundamentales -información y consentimiento- y generó una doctrina que ha excedido con creces a la cultura norteamericana en que nació:

- "A physician violates his duty to his patient and subjects himself to liability if he withholds any facts which are necessary to form the basis of an intelligent consent by the patient to the proposed treatment....At the same time, the physician must place the welfare of his patient above all else and [recognize] that the patient's mental and emotional condition is important and in certain cases may be crucial, and that in discussing the element of risk, a certain amount of discretion must be employed consistent with the full disclosure of facts necessary to an informed consent".

Truman v. Thomas (1982).

Los hijos de una mujer muerta por cáncer cervicouterino ganaron una demanda contra el médico que no informó de los riesgos que corría al omitir su examen de rutina. Es decir, las obligaciones médicas se extienden a la obligación de informar sobre las consecuencias de rechazar intervenciones sanitarias.

- "It must be remembered that Dr. Thomas was not engaged in an arms-length transaction with Mrs. Truman. Clearly $[\ldots]$ he was obligated to provide her with all the information material to her decision [not to have a pap smear]".

\section{Concepto Actual}


Hoy se entiende que es "un proceso", una actividad humana ordenada en el tiempo, que contiene elementos de entrada, actividades o tareas características y productos o resultados.

Un proceso es un conjunto de actividades loǵicamente interrelacionadas y ordenadas que actuán sobre unas entradas y que van a generar un resultado preestablecido para unos usuarios identificados. Consiste en unas entradas, unas tareas que se realizan sobre esas entradas, a las que anãden valor y producen un resultado (7). Ese "proceso de Consentimiento Informado" es propio de la buena práctica médica, que forma parte del contrato civil entre un médico y su paciente, que es requisito absoluto para tratar de extinguir el riesgo de una acción penal por parte del Estado, que es exigible por la autoridad administrativa a las instituciones sanitarias en que se desarrollan actividades de riesgo, y que -en ciertos casos- debe ser demostrado por escrito, cumpliendo ciertos mínimos en la calidad de la información entregada a los pacientes.

Sus componentes esenciales son:

1.- Que toda persona tiene derecho a ser informada, en forma oportuna y comprensible (adecuada a su idioma y nivel cultural), por parte del médico u otro profesional tratante, acerca del estado de su salud, del posible diagnóstico de su enfermedad, de las alternativas de tratamiento disponibles para su recuperación (descripción y propósito) y de los riesgos (los más frecuentes y los más graves) que ello pueda representar, así como del pronóstico esperado, y del proceso previsible del posoperatorio cuando procediere, de acuerdo con su edad y condición personal y emocional. Del mismo modo cabría agregar expresamente las alternativas posibles, limitaciones personales o institucionales para ofrecer otras alternativas, y la posibilidad de revocar el consentimiento, sin expresión de causa.

2.- Que toda persona tiene derecho a prestar su autorización, observaciones o rechazo expreso frente a intervenciones sanitarias potencialmente peligrosas (personalmente o representado).

3.- Que todo prestador sanitario tiene el deber de obtener y conservar los medios de prueba necesarios para demostrar que ha respetado esos derechos de sus pacientes en ciertas condiciones definidas por la ética, la costumbre y la ley.

Por regla general, este proceso se efectuará en forma verbal, pero deberá constar por escrito en el caso de intervenciones quirúrgicas, procedimientos diagnósticos y terapéuticos invasivos y, en general, para la aplicación de procedimientos que conlleven un riesgo relevante y conocido para la salud del afectado. En estos casos, tanto la información misma, como el hecho de su entrega, la aceptación o el rechazo deberán constar por escrito en la fícha clínica del paciente y referirse, al menos, a los contenidos mínimos ya mencionados.

Desde el punto de vista profesional, es imprescindible comprender que:

1.- Ha sido repetidamente invocado en sentencias hispanoparlantes desde el año 1994, aunque el texto original es del Tribunal Supremo de España, ha sido acogido con rigor por tribunales chilenos(8).

2.- Goza de reconocimiento internacional, como refleja un documento de consenso publicado en el año 2002(9).

3.- Se trata de una obligación profesional, contenida en el Código de Ética vigente del Colegio Médico de Chile, la versión editada en 2019(10).

4.- Forma parte del ordenamiento jurídico nacional desde la promulgación de la ley 20.584, que regula los derechos y deberes de las personas en relación con acciones vinculadas a su atención de salud, la cual entró en vigencia el 1 de octubre de 2012(11).

\section{Escenario: Anestesia Regional}

El incremento del uso de anestesia y/o analgesia regional en las últimas décadas ha despertado gran interés en sus aspectos éticos y médico legales. La práctica de técnicas de AR implica la ejecución de procedimientos con riesgos específicos, si bien infrecuentes, potencialmente catastróficos para el paciente (12).

El consentimiento informado no sólo es la base médico legal y ética de un procedimiento médico, también representa una oportunidad de afianzar la relación médico paciente. Entre los fundamentos y principios de un consentimiento informado se encuentra el respeto por la autonomía del paciente, es decir, su derecho de tomar decisiones acerca del manejo médico, de forma voluntaria, informada, conociendo riesgos, beneficios y alternativas de tratamiento. Así como también su derecho de rechazar o declinar un tratamiento(13). Un adecuado proceso de consentimiento permite construir una relación de confianza entre el paciente y el anestesiólogo, donde ambas partes tienen la posibilidad de expresar y aclarar expectativas y dudas. 
Un estudio observacional prospectivo realizado en Reino Unido, evaluó en pacientes sometidos a artroplastía de hombro cuánto recordaban y entendían del CI anestésico v/s el quirúrgico. Observaron que el recuerdo de los riesgos quirúrgicos era significativamente mayor que el recuerdo de los riesgos relacionados al bloqueo de plexo braquial. Un tercio de los pacientes no consideraba la discusión sobre los riesgos de la anestesia regional tan importante como el CI para la cirugía(14). Otro estudio realizado en España, de un total de 159 pacientes que firmaron un CI anestésico, un $64 \%$ lo recordaba vagamente o no lo recordaba(15). Las razones que explicarían estos hallazgos son multifactoriales; en la práctica anestésica el contacto con el paciente es frecuentemente limitado a un solo encuentro, por un corto período de tiempo en el preoperatorio inmediato. Esto plantea la necesidad de mejorar la documentación del consentimiento y la comprensión de éste, implementando medidas que permitan asegurar una discusión de calidad entre anestesiólogo y paciente.

\section{Dificultades en el proceso}

Waisel et al(16) realizaron un análisis cualitativo de dificultades y/o conflictos a las que se enfrenta el anestesiólogo al momento de obtener un CI. Ellos identificaron tres tipos de desafíos: éticos, prácticos y relacionales.

Los desafíos éticos están relacionados con el respeto por los deseos del paciente y su autonomía para discernir; cuando existe conflicto entre los deseos del paciente/familia y el juicio médico; cuando el anestesiólogo debe definir la capacidad de toma de decisiones de un paciente y su habilidad para comprender la información. Los desafíos prácticos se refieren a la cantidad de información que debería ser entregada, a barreras de comunicación como diferencias idiomáticas o un paciente somnoliento porque recibió sedación previo al proceso del consentimiento y limitaciones de tiempo ya comentadas. Y finalmente los desafíos relacionales, que se refieren al cuestionamiento sobre la competencia del anestesiólogo, por ejemplo: un profesional joven podría proyectar falta de experiencia, lo que el paciente percibe como incompetencia, experiencias anestesiológicas negativas previas podrían generar aprehensiones y desconfianza. Un paciente/familia con mucha ansiedad, irritable y demandante fue descrito como un desafío adicional para el anestesiólogo. Lo anterior plantea la necesidad de entregar herramientas de comunicación específicas al proceso de CI a los residentes de anestesiología como un objetivo en su formación, con el fin de mejorar la entrega de información y hacer más eficiente el proceso.

Tabla 1

\section{Fundamentos de un CI de AR}

En nuestro país no existe un proceso formal y estandarizado de CI de AR y analgesia ambulatoria/domiciliaria, incluso el CI genérico de anestesia presenta una gran variación entre los centros de salud particularmente en relación al formulario escrito. Es necesario confeccionar un documento que permita brindar información fidedigna y de calidad a los pacientes y un marco de respaldo médico legal para los anestesiólogos. Esto último cobra relevancia en este período de pandemia, en que el reinicio de la actividad quirúrgica contempla priorizar la cirugía ambulatoria(2). Se requerirá una estrategia de manejo analgésico que permita una rehabilitación y alta temprana, eventualmente analgesia domiciliaria, para ello, los expertos recomiendan AR como una buena alternativa(2-4).

Las recomendaciones nacionales e internacionales sugieren preferir anestesia regional (AR) para anestesia y analgesia por sobre anestesia general $(3,4)$. Tanto la AR neuroaxial como periférica tienen mejor relación riesgo/beneficio(3). La AR disminuye la exposición del personal de salud a procedimientos con riesgo de aerosolización del virus, reduce el consumo de drogas que prolongan la recuperación, brinda un óptimo manejo del dolor agudo postoperatorio y permite analgesia domiciliaria en el caso de bloqueos periféricos continuos. Todo lo anterior conlleva a una rápida recuperación, deambulación y alta hospitalaria precoz(3).

El proceso de CI para AR es mucho más que un mero requisito legal, involucra además de la firma de formularios la discusión de las técnicas anestésicas $(17,18)$. El médico debe asegurar que se discutan y comprendan a cabalidad los riesgos y beneficios del bloqueo, responder preguntas y aclarar dudas $(3,14,19)$.

\section{Items básicos a discutir}

Definir riesgos es fundamental a la hora de discutir el CI de una técnica regional. Lamentablemente es difícil cuantificar las tasas de incidencia de eventos relativamente raros, incluso con extensos estudios de cohorte. Además, los riesgos son específicos de cada técnica o bloqueo y de cada paciente(13).

En el proceso de CI de AR se debe intentar una balanceada y precisa discusión sobre los beneficios y riesgos. Si se entrega extensa información sobre los riesgos asociados a AR minimizando los beneficios, podría influenciar al 
paciente a optar por anestesia general y viceversa si los riesgos de la anestesia general son enfatizados. Esta forma de influenciar la decisión del paciente enfatizando los efectos negativos ha sido denominada comunicación nociva(20).

Existe gran variabilidad en el tipo y cantidad de información que se entrega al paciente(21). Brull et al.(22) realizó una encuesta a anestesiólogos de la Sociedad Americana de Anestesia Regional (ASRA) sobre complicaciones de técnicas de AR. Un 66\% de los encuestados dice discutir riesgos y beneficios con la intención de que el paciente pueda tomar una decisión informada, mientras que un $28 \%$ lo hace por razones médico legales. Para bloqueos neuroaxiales y/o periféricos los riesgos más frecuentemente informados al paciente son cefalea y dolor local/discomfort, mientras que complicaciones neurológicas más severas como neuropatía permanente son inconsistentemente discutidas. Las complicaciones menos discutidas fueron convulsiones, falla respiratoria, para cardio respiratorio y muerte. Es decir, los riesgos en AR que se discuten con mayor frecuencia son los considerados "benignos"(22).

Por otra parte, existen elementos que dificultan aún más la cuantificación del riesgo; escasa claridad en cómo se define una complicación, cuán largo fue el seguimiento de los pacientes después de una complicación para evaluar la resolución de ésta, factores relacionados al paciente que podrían incrementar su riesgo, cuándo una complicación es directamente de causa anestésica o por factores quirúrgicos como la posición o el uso de torniquete de isquemia(13).

En las Guías ASRA 2015 sobre complicaciones neurológicas asociadas a AR(12), se describe que la neuropatía periférica ocurre en $0-2,2 \%$ de los pacientes a 3 meses, $0-0,8 \%$ a los 6 meses y $0-0,2 \%$ al año. Mientras que la incidencia global de síntomas sugerentes de daño de nervio periférico a largo plazo, reportada por las Guías ASRA 2016(23) fue de 5 en 10.000 pacientes.

La Asociación de Anestesiólogos de Gran Bretaña e Irlanda, publicó en 2017(19) guías para una apropiada discusión y documentación del CI anestésico. La Sociedad de Anestesia del Reino Unido(24) ratifica estas recomendaciones que incluyen plantear:

- Beneficios del procedimiento (deambulación temprana, menos náuseas y vómitos, etc).

- Proceso (uso de ultrasonografía, monitorización, etc)

- Alternativas a AR (sedación, anestesia general)

- Educación (protección de la extremidad insensible, recuperación del bloqueo, etc)

- Eventos comunes y complicaciones más importantes del procedimiento reportados en la literatura.

Para tener un estándar de discusión y evitar la variabilidad en la entrega de información. Expertos recomiendan clasificar los riesgos según frecuencia(19):

- Muy comunes- 1:10

- Comunes - entre 1:10 y 1:100

- Poco comunes - entre 1:100 y 1:1.000

- Raros - entre 1:1000 Y 1:10.000

- Muy raros: mayor de 1:10.000

\section{Consideraciones en analgesia domiciliaria}

Bloqueos continuos de nervio periférico (BCNP) son una técnica analgésica efectiva en el postoperatorio, aumenta la satisfacción del paciente, mejoran los outcomes quirúrgicos, aceleran la rehabilitación y permiten un manejo domiciliario del paciente.

Los riesgos asociados a BCNP son raros $(0-9,7 \%)(12,25,26)$. Bryan et al. en una serie de 144 pacientes con catéter interescalénico para cirugía de hombro, describió una tasa de complicaciones de $0.7 \%(25)$.

En el caso de CI que involucre BCNP para analgesia domiciliaria, además de la discusión del procedimiento y los riesgos inherentes al bloqueo, se deben explicitar complicaciones tales como: analgesia inadecuada, readmisión, retiro accidental del catéter o desconexión, entre otras(27).

\section{Estructura de un proceso de CI de buena calidad}

Seguń la norma ISO 9000:2000 un proceso es "un conjunto de actividades mutuamente relacionadas o que interactuán, las cuales transforman elementos de entrada en resultados". Con esta definicioń, se puede deducir que el enfoque basado en procesos enfatiza coḿo los resultados que se desean obtener se pueden alcanzar de manera maś eficiente considerando las actividades agrupadas entre sí. 
La palabra proceso viene del latín processus, que significa avance y progreso(28).

Un proceso es el conjunto de actividades de trabajo interrelacionadas que se caracterizan por requerir ciertos insumos (inputs: productos o servicios obtenidos de otros proveedores) y tareas particulares que implican valor anãdido, con miras a obtener ciertos resultados. Una definicioń alternativa indica: gestioń de todas las actividades de la empresa que generan un valor anãdido; o bien, conjunto de actividades mutuamente relacionadas o que interactuán, las cuales transforma elementos de entrada en resultados.

Proceso no es lo mismo que procedimiento. Un procedimiento es el conjunto de reglas e instrucciones que determinan la manera de proceder o de obrar para conseguir un resultado. Un proceso define que es lo que se hace, y un procedimiento, coḿo hacerlo.

No todas las actividades que se realizan son procesos. Para determinar si una actividad realizada por una organizacioń es un proceso o subproceso, debe cumplir los siguientes criterios:

1.- La actividad tiene una misión o propósito claro.

2.- La actividad contiene entradas y salidas, se pueden identificar los clientes, proveedores y producto final.

3.- La actividad debe ser susceptible de descomponerse en operaciones o tareas.

4.- La actividad puede ser estabilizada mediante la aplicación de la metodología de gestión por procesos (tiempo, recursos, costes).

\section{5.- Se puede asignar la responsabilidad del proceso a una persona.}

El proceso de Consentimiento Informado puede alcanzar complejidades inesperadas, porque el operador que indica la actividad de riesgo puede ser ciego a los riesgos que conocen los especialistas que la despliegan.

En ese caso, el rol del prestador institucional se hace distinto y superior al de los prestadores individuales, que pueden responder como operadores institucionales cuando indican y despliegan actividades de riesgo (con la carga inevitable de que, en esas condiciones, pueden enfrentar el baldón del incentivo pecuniario que podría sesgarlos).

A manera de ejemplo, la decisión de una resonancia nuclear magnética bajo anestesia general implica -al menos- la participación de tres actores diferentes y especializados, que se deben coordinar para brindar la mejor eficiencia y seguridad a cada paciente: el especialista que la indica con propósitos diagnósticos, el radiólogo que mejor conoce los requerimientos de tiempos, apneas y uso de gadolinio que pueden ser necesarios o inevitables, y el anestesista que debe enfrentar las condiciones especiales propias de una anestesia fuera de pabellón en ese entorno altamente magnético.

En el caso de las anestesias regionales se deben coordinar: el especialista que indica o ejecuta una posible intervención dolorosa en un escenario de pandemia, el anestesista que puede considerar los beneficios ofrecidos por la anestesia regional y bloqueos de nervios periféricos, y las personas que podrían estar encargadas del cuidado posoperatorio inmediato y rehabilitación diferida de un individuo que puede tener muchas peculiaridades de personalidad y de estilo de vida, además de la enfermedad que la lleva a requerir auxilio sanitario.

Desde esa perspectiva, el proceso de Consentimiento Informado debería considerar, al menos:

1.- Misión o propósito: Cumplir normas éticas y legales que regulan el quehacer médico en Chile. Ese objetivo implica poner a los pacientes que podrían enfrentar intervenciones sanitarias de riesgo en posición de decidir libremente de acuerdo a sus intereses y expectativas sobre su propia vida. El mismo objetivo general implica dejar constancia de ello en una manera que sea admisible en una revisión por la autoridad administrativa o judicial.

2.- Entradas: Pacientes que pueden enfrentar intervenciones de riesgo. Salidas: Pacientes bien informados sobre las intervenciones propuestas, y documentos probatorios de lo que ellos aceptaron, condicionaron o rechazaron en esas condiciones de buena información.

3.- Operaciones: Generación de documentos institucionales (de información y de constancia) y selección de pacientes; entrega de información inicial y protocolo de derivación expedita a Unidades de Apoyo; plazo razonable para comprensión, reflexión y administración de dudas y conflictos; resolución por acuerdo entre el prestador y el paciente; documentación del acuerdo. 
4.- Responsabilidades: Operador que indica la intervención sanitaria que implica riesgos (información inicial, registro y derivación); operadores que podrían desplegar la actividad sanitaria riesgosa (información definitiva, acuerdos y registro); personal institucional dedicado a control y registro de documentación (verificación de registros y custodia).

\section{Estructura de un formulario de CI de buena calidad}

Por regla general, el proceso de CI se efectuara' en forma verbal, pero debera' constar por escrito en el caso de intervenciones quirurgicas, procedimientos diagnośticos y terapeúticos invasivos y, en general, para la aplicacioń de procedimientos que conlleven un riesgo relevante y conocido para la salud del afectado. En estos casos, tanto la informacioń misma, como el hecho de su entrega, la aceptacioń o el rechazo deberań constar por escrito en la ficha clínica del paciente y referirse, al menos, a los contenidos indicados en el inciso primero del artículo 10 de la ley 20.584. Se presume que la persona ha recibido la información pertinente para la manifestacioń de su consentimiento, cuando hay constancia de su firma en el documento explicativo del procedimiento o tratamiento al cual deba someterse(29).

En primer lugar, y aunque parezca obvio, cabe mencionar algunos requisitos formales y de interpretación que están consagrados en el ordenamiento jurídico chileno, específicamente en la "Ley de Protección al Consumidor": el texto debe estar en castellano (por extensión, en el idioma del paciente), las cláusulas particulares priman sobre las generales, los espacios en blanco deben ser llenados o inutilizados antes de la suscripción del documento, y el tamaño de las letras impresas no puede ser inferior a $2,5 \mathrm{~mm}(30)$.

En segundo lugar cabe citar los requisitos generales de la información exigidos por la "Ley de Derechos y Deberes de los Pacientes" en el ya mencionado Art. 10: "Toda persona tiene derecho a ser informada, en forma oportuna y comprensible, por parte del medico u otro profesional tratante, acerca del estado de su salud, del posible diagnośtico de su enfermedad, de las alternativas de tratamiento disponibles para su recuperacioń y de los riesgos que ello pueda representar, asícomo del pronośtico esperado, y del proceso previsible del postoperatorio cuando procediere, de acuerdo con su edad y condicion personal y emocional".

En tercer lugar se deberían agregar las informaciones requeridas por la jurisprudencia citada en sentencias nacionales, sobre las obligaciones de información imputables al acto médico: "B) Informar al paciente o, en su caso, a los familiares del mismo, siempre, claro esta, que ello resulte posible, del diagnośtico de la enfermedad o lesioń que padece, del pronośtico que de su tratamiento puede normalmente esperarse, de los riesgos que el mismo, especialmente si eśte es quiruŕgico, pueden derivarse $\mathrm{y}$, finalmente, $\mathrm{y}$ en el caso de que los medios de que se disponga en el lugar donde se aplica el tratamiento puedan resultar insuficientes, debe hacerse constar tal circunstancia, de manera que, si resultase posible, opte el paciente o sus familiares por el tratamiento del mismo en otro centro medico mas adecuado; [...] y D) En los supuestos -no infrecuentes- de enfermedades o dolencias que puedan calificarse de recidivas (sic), crońicas o evolutivas, informar al paciente de la necesidad de someterse a los analisis y cuidados preventivos y que resulten necesarios para la prevencioń del agravamiento o repeticioń de la dolencia"(31).

\section{Recomendaciones}

- Todo procedimiento médico debe estar respaldado por la discusión y documentación de un consentimiento informado(17,19).

- Evaluación preoperatoria: Esta es la oportunidad de informar y discutir la técnica anestésica, resolver dudas acerca de ella y firmar -eventualmente- el consentimiento del procedimiento (a menos que el paciente reclame un tiempo de reflexión, que debe ser respetado).

- Durante la pandemia por COVID-19, expertos recomiendan realizar evaluación preoperatoria por telemedicina para reducir contagios y mantener una aproximación personal con el paciente(3).

- Expertos sugieren informar detalladamente de manera oral y escrita al paciente y/o su representante de las circunstancias específicas relacionadas a la pandemia por COVID-19. En particular, información acerca de la relación riesgo/beneficio del procedimiento. El mensaje debe ser claro, objetivo y basado en la mejor evidencia disponible. Lo que debe quedar registrado en la ficha médica del paciente(3).

- Expertos recomiendan utilizar un cuestionario estandarizado con el objetivo de evaluar la presencia de síntomas compatibles con infección SARS-CoV-2 antes del procedimiento(2,3). Este documento debe adjuntarse al CI.

- Asegurar un espacio físico adecuado, tranquilo, considerar tiempo suficiente para una discusión a cabalidad del CI(19). 
- Para facilitar la discusión de los puntos discutidos durante el proceso de CI, se recomienda incorporar y entregar a los pacientes folletos o ayudas cognitivas con la información relevante(13).

- La responsabilidad del CI en AR recae en el anestesiólogo que realiza el bloqueo(13).

- Para analgesia domiciliaria documentar riesgos relacionados al cuidado y manejo del catéter de nervio periférico(27).

\section{Referencias}

1. World Health Organization W. WHO Director-General's opening remarks at the media briefing on COVID-19 - 11 March 2020 [Internet]. WHO Director General's speeches. 2020 [cited 2020 Sep 5]. p. 4. Available from: https://www.who.int/dg/speeches/detail/who-director-general-s-opening-remarks-at-the-media-briefing-oncovid-19---11-march-2020

2. Aranda F, Aliste J, Altermatt F, Alvarez JP, Bernucci F, Cabrera MC, et al. Recomendaciones para el manejo de pacientes con COVId19 en el perioperatorio. Revista Chilena de Anestesia. 2020;49(2):196-202.

3. Velly L, Gayat E, Quintard H, Weiss E, de Jong A, Cuvillon P, et al. Guidelines: Anaesthesia in the context of COVID-19 pandemic. Anaesthesia Critical Care \& Pain Medicine [Internet]. 2020 Jun;39(3):395-415. Available from: https://linkinghub.elsevier.com/retrieve/pii/S2352556820300977

4. Aliste J, Altermatt F, Atton R, Bravo D, Layera S, Miranda P, et al. Recomendaciones para la ejecución de anestesia regional no obstétrica en perioperatorio de pacientes COVID-19. Revista Chilena de Anestesia [Internet]. 2020 [cited 2020 Sep 5];49(3). Available from: https://revistachilenadeanestesia.cl/revchilanestv49n03-08/

5. Mutizábal G. Consentimiento Informado y Anestesia. Revista Chilena de Anestesia [Internet]. 2011 [cited 2020 Sep 5];40(1):77-102. Available from: https://revistachilenadeanestesia.cl/consentimiento-informado-y-anestesial

6. Mutizábal G. Consentimiento informado en investigación. Revista Chilena de Anestesia. 2014;43(4):368-408.

7. Guías integradas asistenciales. Metodología para la estandarización de actividades basadas en la calidad y en los sistemas de clasificación de pacientes GRD. Instituto Nacional de Salud, Subdirección General de Coordinación Administrativa. Madrid [Internet]. Guía. [cited 2020 Sep 5]. Available from: https:// ingesa.sanidad.gob.es/bibliotecaPublicaciones/publicaciones/internet/docs/guias/ntegradas.pdf

8. Sentencia Recurso de Casación 349-1994, de 25 de abril de 1994. Tribunal Supremo de España, Sala de lo Civil [Internet]. [cited 2020 Sep 5]. Available from: http://www.poderjudicial.es/search/AN/openDocument/ d9799a535367fcef/20040618

9. Medical Professionalism In The New Millennium: A Physician Charter. [cited 2020 Sep 5]; Available from: https://abimfoundation.org/wp-content/uploads/2015/12/Medical-Professionalism-in-the-New-Millenium-APhysician-Charter.pdf

10. Colegio Médico de Chile. Código de Ética [Internet]. [cited 2020 Sep 5]. Available from: http:// www.colegiomedico.cl/wp-content/uploads/2019/12/codigo-de-etica_FINAL.pdf

11. Ley-20583 [Internet]. [cited 2020 Sep 5]. Available from: https://www.bcn.cl/leychile/navegar? $\underline{\text { idNorma }=1039348}$

12. Neal JM, Barrington MJ, Brull R, Hadzic A, Hebl JR, Horlocker TT, et al. The Second ASRA Practice Advisory on Neurologic Complications Associated With Regional Anesthesia and Pain Medicine. Regional Anesthesia and Pain Medicine [Internet]. 2015;40(5):401-30. Available from: https://rapm.bmj.com/lookup/doi/10.1097/ AAP.0000000000000286

13. Tierney S, Perlas A. Informed consent for regional anesthesia. Current opinion in anaesthesiology. 2018;31(5):614-21.

14. Zarnegar R, Brown MRD, Henley M, Tidman V, Pathmanathan A. Patient perceptions and recall of consent for regional anaesthesia compared with consent for surgery. Journal of the Royal Society of Medicine. 2015;108(11):451-6. 
15. Rosique I, Pérez-Cárceles MD, Romero-Martín M, Osuna E, Luna A. The use and usefulness of information for patients undergoing anaesthesia. Medicine and Law [Internet]. 2006 Dec 1 [cited 2020 Sep 5];25(4):715-27. Available from: https://europepmc.org/article/med/17263037

16. Waisel DB, Lamiani G, Sandrock NJ, Pascucci R, Truog RD, Meyer EC. Anesthesiology trainees face ethical, practical, and relational challenges in obtaining informed consent. Anesthesiology. 2009;110(3):480-6.

17. Koyfman SA, Reddy CA, Hizlan S, Leek AC, Kodish and ED. Informed consent conversations and documents: A quantitative comparison. Cancer [Internet]. 2016 Feb 1;122(3):464-9. Available from: http:// doi.wiley.com/10.1002/cncr.29759

18. Lin CJ, Luke C, Sullivan D, Kidwell R. Development of specific peripheral nerve block consent form. Journal of Clinical Anesthesia. 2019;54(September 2018):33-6.

19. Yentis SM, Hartle AJ, Barker IR, Barker P, Bogod DG, Clutton-Brock TH, et al. AAGBI: Consent for anaesthesia 2017. Anaesthesia [Internet]. 2017 Jan;72(1):93-105. Available from: http://doi.wiley.com/10.1111/anae.13762

20. Cyna AM, Simmons SW. Guidelines on informed consent in anaesthesia: Unrealistic, unethical, untenable.. British Journal of Anaesthesia. 2017;119(6):1086-9.

21. Brull R, McCartney CJL, Chan VWS, Liguori GA, Hargett MJ, Xu D, et al. Disclosure of Risks Associated With Regional Anesthesia: A Survey of Academic Regional Anesthesiologists. Regional Anesthesia and Pain Medicine. 2007;32(1):7-11.

22. Brull R, Wijayatilake DS, Perlas A, Chan VWS, Abbas S, Liguori GA, et al. Practice Patterns Related to Block Selection, Nerve Localization and Risk Disclosure: A Survey of the American Society of Regional Anesthesia and Pain Medicine. Regional Anesthesia and Pain Medicine [Internet]. 2008 Sep [cited 2020 Sep 5];33(5):395-403. Available from: https://pubmed.ncbi.nlm.nih.gov/18774508/

23. Neal JM, Brull R, Horn JL, Liu SS, McCartney CJL, Perlas A, et al. The second American society of regional anesthesia and pain medicine evidence-based medicine assessment of ultrasound-guided regional anesthesia: Executive summary. Regional Anesthesia and Pain Medicine. 2016;41(2):181-94.

24. RAUK. RAUK Consent for peripheral nerve blocks 2015. 2015.

25. Bryan NA, Swenson JD, Greis PE, Burks RT. Indwelling interscalene catheter use in an outpatient setting for shoulder surgery: Technique, efficacy, and complications. Journal of Shoulder and Elbow Surgery. 2007;16(4):38895.

26. Capdevila X, Pirat P, Bringuier S, Gaertner E, Singelyn F, Bernard N, et al. Continuous Peripheral Nerve Blocks in Hospital Wards after Orthopedic Surgery. Anesthesiology. 2005;103(5):1035-45.

27. Gallay SH, Lobo JJA, Baker J, Smith K, Patel K. Development of a regional model of care for ambulatory total shoulder arthroplasty: A pilot study. Clinical Orthopaedics and Related Research. 2008;466(3):563-72.

28. La Gestión por Procesos [Internet]. Toledo; 2002 [cited 2020 Sep 5]. Available from: https://www.chospab.es/ calidad/archivos/Documentos/Gestiondeprocesos.pdf

29. Ley 20.584. Regula los derechos y deberes que tienen las personas en relación con acciones vinculadas a su atención en salud [Internet]. [cited 2020 Sep 5]. Available from: https://www.minsal.cl/sites/default/files/files/Ley \%2020584\%20Derechos\%20y\%20Deberes.pdf

30. LEY 19496. Establece normas sobre protección de los derechos de los consumidores [Internet]. [cited 2020 Sep 5]. Available from: https://www.bcn.cl/leychile/navegar?idNorma=61438\&idVersion=2019-09-14\&idParte=

31. Sentencia Recurso de Casación 349-1994, de 25 de abril de 1994. Tribunal Supremo de España, Sala de lo Civil [Internet]. [cited 2020 Sep 5]. Available from: http://www.poderjudicial.es/search/AN/openDocument/ d9799a535367fcef/20040618 
Desafíos para el anestesiólogo en la obtención del consentimiento Informado

\section{Desafíos Éticos}

Respetar los deseos y autonomía del paciente

Conflicto del paciente con el juicio médico

Capacidad del paciente para tomar decisiones

Desafíos Prácticos

Cantidad de información a entregar

Barreras de comunicación

Limitaciones de tiempo

Desafíos Relacionales

Cuestionamiento de la competencia del anestesiólogo

Desconfianza

Falta de precisón y/o claridad en la información 